\section{NEW POLYETHER ANTIBIOTICS, A-130B AND A-130C}

Sir :

Recently we have found two new antibacterial components $\mathrm{A}-130 \mathrm{~B}$ (1) and $\mathrm{C}(2)$ in the metabolites of Streptomyces hygroscopicus A-130, which produces polyether antibiotic A-130A (3). This antibiotic was isolated and characterized as a new polyether antibiotic by КUвотA, et al. ${ }^{1)}$ and later isolated also by an American group as Ro 21-6150*, which was identified with our specimen $^{2 \mathrm{a}}$.

Two active minor components, $\mathbf{1}$ and $\mathbf{2}$, found in the mother liquor of $\mathbf{3}$ were isolated by precise t.l.c. on silica gel. Like 3, both compounds were easily assumed to be polyether antibiotics having an $\alpha, \beta$-unsaturated ketone function from their IR $\left(1655 \mathrm{~cm}^{-1}\right)$ and $\operatorname{UV}\left(\lambda_{\max } 235 \mathrm{~nm}\right)$ spectra (Fig. 1). Up to date, two antibiotics, dianemycir ${ }^{4)}$ and $\mathbf{3}$ are known to belong to this class, but both $\mathbf{1}$ and $\mathbf{2}$ are distinguishable from these antibiotics by IR and t.l.c.

Antibiotics 1 and $\mathbf{2}$ did not give any crystals suitable for X-ray analyses, while the ${ }^{13} \mathrm{C}$ NMR spectra of their sodium salts $(1-\mathrm{Na})$ and $(2-\mathrm{Na})$ gave valuable information in comparison with that of 3-Na, whose structure is established by an X-ray diffraction of $3-\mathrm{Ag}^{2}$. The ${ }^{13} \mathrm{C}$ NMR spectra were examined in $\mathrm{C}_{6} \mathrm{D}_{6}$ and $\mathrm{CDCl}_{3}$, and the signals were assigned by the usual procedures including various ${ }^{1} \mathrm{H}$ decoupling techniques $^{5)}$ and comparison with those of sodium salts of A$28695^{6)}(\text { septamycin })^{7)}(4), \mathrm{K}-$ $41 \mathrm{~A}(5)^{8)}$ and $\mathrm{B}(6)^{9)}$; their ${ }^{13} \mathrm{C}$ NMR signals were already assigned $^{9,10}$.

As shown in Tables 1 and 2, $\mathbf{1}$ is composed of 54 carbons while 2 as well as 3 has 47 carbons. The precise comparison of the spectra led to the assumption that $\mathbf{1}$ has an additional deoxysugar

* Anteunis et al., who studied the ${ }^{1} \mathrm{H}$ NMR spectra of Ro 21-61.50 provided by this group, called it lenoremycin ${ }^{3)}$.
(Deo) moiety $\mathrm{C}_{7} \mathrm{H}_{13} \mathrm{O}_{3}$ like as 6 . The presence of two OMe signals in the ${ }^{1} \mathrm{H}$ NMR spectrum of 1-Na (see Fig. 2) supports the speculation, and the ${ }^{13} \mathrm{C}$ signals due to the Deo moiety are easily pointed out as shown in Table 2. The remaining ${ }^{13} \mathrm{C}$ signals of $\mathbf{1}-\mathrm{Na}$ essentially correspond to those of 3 -Na except 7 carbon signals. Marked and slight changes (* and ** in Table 1, respectively) in the ${ }^{13} \mathrm{C}$ chemical shifts were exhibited from $3-\mathrm{Na}$ to $1-\mathrm{Na}$ as $-\mathrm{CH}_{2-} \rightarrow$ $\stackrel{1}{\mathrm{C}} \mathrm{H}-\mathrm{O}-\left(\delta_{\mathrm{C}} 37.2 \rightarrow 82.6\right)$, upfield shifts of two Me signals $(17.5$ and $17.9 \rightarrow 12.9$ and 14.0 , respectively), downfield shifts of two - $\mathrm{C} H$ - signals ( 36.9 and $33.3 \rightarrow 45.4$ and 40.0 , respectively), and slight signal shifts of $-\stackrel{\mathrm{C}}{\mathrm{C}} \mathrm{H}-\mathrm{O}-(73.5 \rightarrow 72.8)$ and $-\mathrm{CH}<{ }_{\mathrm{O}}^{\mathrm{O}}(99.1 \rightarrow 100.3)$. Taking account of the structure of $\mathbf{3}$, these spectral changes can be expected only by locating the second Deo at C-27 (in ring E). Similar changes were also observed between 4-Na and 5-Na (see Table 3). Further, the $\delta_{\mathrm{C}}$ values for the Deo moiety at C-27 in 1-Na are in good accordance to those of $5-\mathrm{Na}$ (see Table 2). Therefore, the second Deo of $\mathbf{1}$ should be situated just as that of $\mathbf{5}$, and A-130B has been determined as structure $\mathbf{1}^{*}$. (* next page)
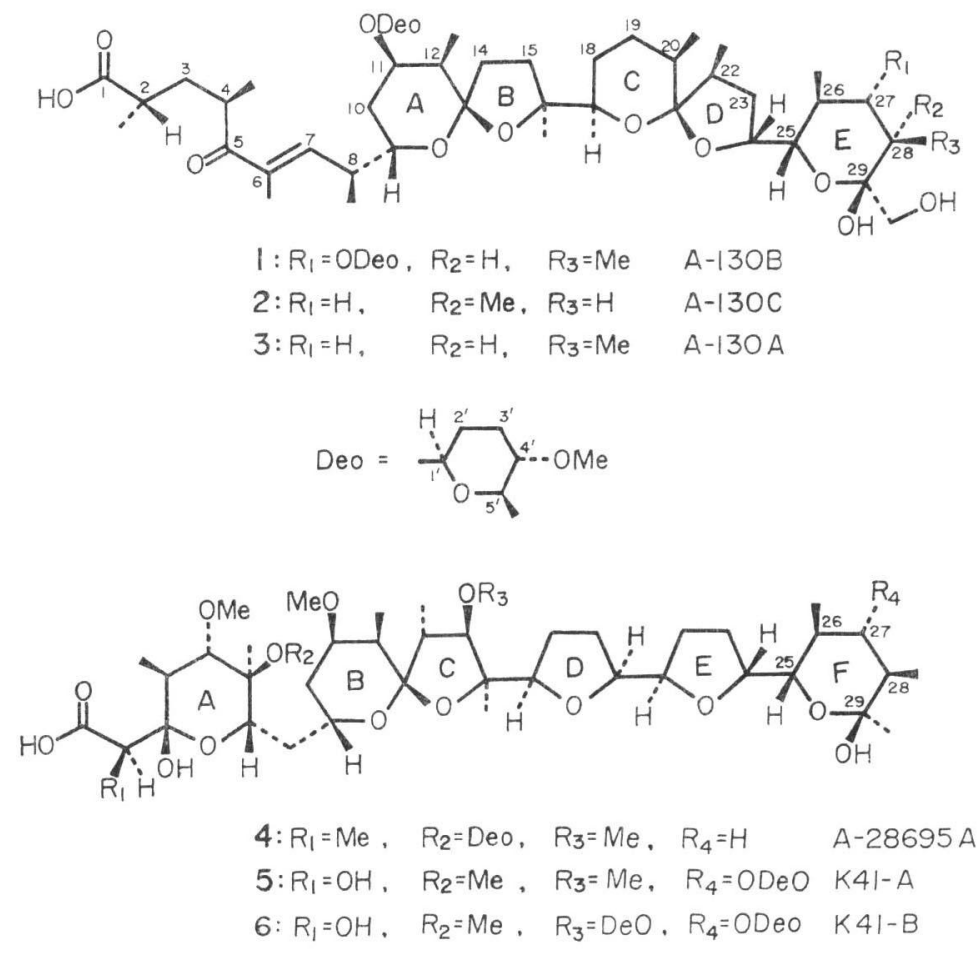
Table 1. ${ }^{13} \mathrm{C}$ Chemical shifts $(\delta)^{\mathrm{a}}$ of A-130A-Na (3-Na), A-130B-Na (1-Na) and A-130C-Na (2-Na).

\begin{tabular}{|c|c|c|c|c|}
\hline $\begin{array}{c}\text { Carbon } \\
\text { type }^{b}\end{array}$ & $\begin{array}{l}\text { Assign- } \\
\text { ment }^{\mathrm{c}}\end{array}$ & $3-\mathrm{Na}$ & $1-\mathrm{Na}$ & $2-\mathrm{Na}$ \\
\hline $\mathrm{C}-\mathrm{Me}(\mathrm{q})$ & $\begin{array}{l}2-\mathrm{Me}^{\mathrm{d}} \\
4-\mathrm{Me}^{\mathrm{d}} \\
6-\mathrm{Me}^{-} \\
8-\mathrm{Me}^{\mathrm{d}} \\
12-\mathrm{Me} \\
16-\mathrm{Me} \\
20-\mathrm{Me}^{\mathrm{d}} \\
22-\mathrm{Me}^{\mathrm{d}} \\
26-\mathrm{Me} \\
28-\mathrm{Me}\end{array}$ & $\begin{array}{l}20.9 \\
17.7 \\
11.7 \\
14.3 \\
13.9 \\
27.3 \\
15.0 \\
15.3 \\
17.9 \\
17.5\end{array}$ & $\begin{array}{l}20.8 \\
17.7 \\
11.7 \\
14.2 \\
13.9 \\
27.4 \\
15.1 \\
15.2 \\
14.0^{*} \\
12.9^{*}\end{array}$ & $\begin{array}{l}20.9 \\
17.7 \\
11.8 \\
14.3 \\
13.9 \\
27.6 \\
15.1 \\
15.3 \\
18.3^{* *} \\
15.5^{*}\end{array}$ \\
\hline $\begin{array}{c}\mathrm{C}-\mathrm{CH}_{2}- \\
(\mathrm{t})\end{array}$ & $\begin{array}{l}\text { C-3 } \\
\text { C-10 } \\
\text { C-14 } \\
\text { C-15 } \\
\text { C-18 } \\
\text { C-19 } \\
\text { C-23 } \\
\text { C-27 }\end{array}$ & $\begin{array}{l}41.9 \\
29.8^{\mathrm{e}} \\
36.0 \\
32.5 \\
17.7 \\
27.6^{\mathrm{f}} \\
36.7 \\
37.2\end{array}$ & $\begin{array}{l}41.8 \\
29.7 \mathrm{e} \\
36.0 \\
32.4 \\
17.7 \\
27.4^{\mathrm{r}} \\
36.7 \\
-\end{array}$ & $\begin{array}{l}41.9 \\
29.6^{\mathrm{e}} \\
36.1 \\
32.5 \\
17.7^{\mathrm{f}} \\
27.6^{\mathrm{f}} \\
36.8^{-} \\
36.1^{\text {** }}\end{array}$ \\
\hline$\underset{\text { (d) }}{\mathrm{C}-\mathrm{CH}}<$ & $\begin{array}{l}\text { C-2 } \\
\text { C-4 } \\
\text { C-8 } \\
\text { C-12 } \\
\text { C-20 } \\
\text { C-22 } \\
\text { C-26 } \\
\text { C-28 }\end{array}$ & $\begin{array}{l}39.8 \\
38.4 \\
41.9 \\
39.8 \\
30.4 \\
35.4 \\
33.3 \\
36.9\end{array}$ & $\begin{array}{l}39.8 \\
38.3 \\
41.8 \\
39.8 \\
30.3 \\
35.4 \\
40.0^{*} \\
45.4^{*}\end{array}$ & $\begin{array}{l}39.8 \\
38.4 \\
41.9 \\
39.9 \\
30.4 \\
35.8 \\
26.3^{*} \\
36.4^{* *}\end{array}$ \\
\hline $\begin{array}{l}\mathrm{O}-\mathrm{CH}_{2-}^{-} \\
(\mathrm{t})\end{array}$ & $C-30$ & 64.7 & 64.6 & 65.0 \\
\hline $\begin{array}{l}\mathrm{O}-\mathrm{CH}< \\
\text { (d) }\end{array}$ & $\begin{array}{l}\text { C-9 } \\
\text { C-11 } \\
\text { C-17 } \\
\text { C-24 } \\
\text { C-25 } \\
\text { C-27 }\end{array}$ & $\begin{array}{r}68.5 \\
73.5 \\
80.9 \\
79.6 \\
73.5 \\
-\end{array}$ & $\begin{array}{l}68.5 \\
73.5 \\
80.9 \\
79.8 \\
72.8^{* *} \\
82.6^{*}\end{array}$ & $\begin{array}{l}68.4 \\
73.7 \\
81.0 \\
79.6 \\
74.2^{\text {** }} \\
-\end{array}$ \\
\hline $\mathrm{O}-\mathrm{C}^{\prime}(\mathrm{s})$ & $C-16$ & 85.8 & 85.9 & 85.9 \\
\hline$\stackrel{\mathrm{O}}{\mathrm{O}}>\mathrm{C}<(\mathrm{s})$ & $\begin{array}{l}\mathrm{C}-13 \\
\mathrm{C}-21 \\
\mathrm{C}-29\end{array}$ & $\begin{array}{r}109.3 \\
111.1 \\
99.1\end{array}$ & $\begin{array}{l}109.0 \\
111.3 \\
100.3^{* *}\end{array}$ & $\begin{array}{l}109.1 \\
111.1 \\
98.0 * *\end{array}$ \\
\hline$=\mathrm{CH}-(\mathrm{d})$ & $C-7$ & 146.0 & 145.9 & 145.8 \\
\hline$=\mathrm{C}<\quad(\mathrm{s})$ & C-6 & 134.5 & 134.5 & 134.8 \\
\hline$>\mathrm{C}=\mathrm{O}(\mathrm{s})$ & $\begin{array}{l}\text { C-1 } \\
\text { C-5 }\end{array}$ & $\begin{array}{l}181.3 \\
205.8\end{array}$ & $\begin{array}{l}181.6 \\
205.8\end{array}$ & $\begin{array}{l}181.9 \\
206.0\end{array}$ \\
\hline
\end{tabular}

a ${ }^{13} \mathrm{C}$ FT NMR spectra were recorded on a Varian XL-100-12A spectrometer at $25.160 \mathrm{MHz}$ in $\mathrm{C}_{6} \mathrm{D}_{6}\left(\mathrm{ca} .0 .25 \mathrm{mmol} \cdot \mathrm{cm}^{-3}\right)$ at $35^{\circ} \mathrm{C}$; accuracies of $\delta$ values are $\pm 0.05 \mathrm{ppm}$.

b Multiplicities were obtained by ${ }^{1} \mathrm{H}$ singlefrequency off-resonance decoupling experiments.

c $\delta$ values of Deo carbons are listed in Table 2.

d Tentative assignments.

e ${ }^{\mathrm{f}}$ Assignments may be interchanged (for interchangeable signal, see Table 2).

*, ** See text.
Table 2. ${ }^{13} \mathrm{C}$ Chemical shifts $(\delta)^{\mathrm{a}}$ of the deoxysugar (Deo) moieties of A-130A-Na (3-Na), A-130B-Na (1-Na), A-130C-Na (2-Na), and K-41A-Na (5-Na).

\begin{tabular}{|c|c|c|c|c|c|c|}
\hline \multirow{2}{*}{$\begin{array}{c}\text { Carbon } \\
\text { type }^{\mathrm{b}}\end{array}$} & \multirow{2}{*}{$\begin{array}{c}\text { Assign- } \\
\text { ment }\end{array}$} & \multicolumn{3}{|c|}{ Deo at $C-11^{c}$} & \multicolumn{2}{|c|}{$\begin{array}{c}\text { Deo at } \\
C-27^{d}\end{array}$} \\
\hline & & $3-\mathrm{Na}$ & $1-\mathrm{Na}$ & $2-\mathrm{Na}$ & $1-\mathrm{Na}$ & $5-\mathrm{Na}$ \\
\hline $\mathrm{O}_{(\mathrm{d})}^{\mathrm{O}}>\mathrm{CH}_{-}$ & C- $1^{\prime}$ & 103.3 & 103.2 & 103.5 & 103.2 & 103 \\
\hline $\begin{array}{c}-\mathrm{CH}_{2-} \\
(\mathrm{t})\end{array}$ & C-2' & $28.7^{\mathrm{r}}$ & $28.6^{\mathrm{t}}$ & $28.8^{\mathrm{f}}$ & 30.9 & 31.0 \\
\hline $\begin{array}{c}-\mathrm{CH}_{2}- \\
(\mathrm{t})\end{array}$ & C-3' & $26.6^{\mathrm{e}}$ & $26.5^{\mathrm{e}}$ & $26.7^{\mathrm{e}}$ & 27.4 & 27. \\
\hline$\underset{\text { (d) }}{\mathrm{O}-\mathrm{CH}}$ & C- $4^{\prime}$ & 79.5 & 79.5 & 79.6 & 80.6 & 80.6 \\
\hline $\begin{array}{c}\mathrm{O}-\mathrm{CH}< \\
\text { (d) }\end{array}$ & C- $-5^{\prime}$ & 76.8 & 76.7 & 76.8 & 74.5 & 74. \\
\hline $\mathrm{C}-\mathrm{Me}(\mathrm{q})$ & $5^{\prime}-\mathrm{Me}$ & 18.6 & 18.7 & 18.7 & 18.7 & 18.8 \\
\hline $\mathrm{O}-\mathrm{Me}(\mathrm{q})$ & $4^{\prime}$-OMe & 56.3 & 56.3 & 56.4 & 56.2 & 56. \\
\hline
\end{tabular}

a See footnote a in Table 1.

b See footnote $b$ in Table 1 .

${ }^{\mathrm{c}},{ }^{\mathrm{d}}$ Chemical shift differences found between Deo at C-11 and that at C-27 may be attributable to the environmental difference; Deo at C-11 directly participates in the metal-ion capture in solution ${ }^{11)}$ as well as in crystal ${ }^{2)}$.

${ }^{e}{ }^{\mathrm{f}}$ Assignments may be interchanged (for the interchangable signal, see Table 1).

Table 3. Comparison of ${ }^{13} \mathrm{C}$ data ${ }^{a}$ in ring $\mathrm{E}$ of 1$\mathrm{Na}, 3-\mathrm{Na}$ and in ring $\mathrm{F}$ of $4-\mathrm{Na}$, and $5-\mathrm{Na}$.

\begin{tabular}{l|r|r|r|r|r|r}
\hline & $1-\mathrm{Na}$ & $\begin{array}{c}3- \\
\mathrm{Na}\end{array}$ & $\Delta \delta^{\mathrm{b}}$ & $\begin{array}{c}5- \\
\mathrm{Na}\end{array}$ & $\begin{array}{c}4- \\
\mathrm{Na}\end{array}$ & $\Delta \delta^{\mathrm{c}}$ \\
\hline $\mathrm{C}-25$ & 72.8 & 73.5 & -0.7 & 74.6 & 75.7 & -0.9 \\
$\mathrm{C}-26$ & 40.0 & 33.3 & +6.7 & 39.8 & 33.2 & +6.6 \\
$\mathrm{C}-27$ & 82.6 & 37.2 & +45.4 & 82.9 & 37.5 & +45.4 \\
$\mathrm{C}-28$ & 45.4 & 36.9 & +8.5 & 48.2 & 40.5 & +7.7 \\
$\mathrm{C}-29$ & 100.3 & 99.1 & +1.2 & 98.9 & 97.4 & +1.5 \\
$26-\mathrm{Me}$ & 14.0 & 17.9 & -3.9 & 13.8 & 17.5 & -3.7 \\
$28-\mathrm{Me}$ & 12.9 & 17.5 & -4.6 & 13.2 & 17.4 & -4.2 \\
$29-\mathrm{CH}_{2} \mathrm{OH}$ & 64.6 & 64.7 & -0.1 & - & - & - \\
$29-\mathrm{Me}$ & - & - & - & 27.2 & 26.8 & +0.4 \\
\hline
\end{tabular}

a $\delta$ values in $\mathrm{C}_{6} \mathrm{D}_{6}$.

b Chemical-shift difference; $\Delta \delta=\delta(\mathbf{1}-\mathrm{Na})-\delta(\mathbf{3}-$ $\mathrm{Na})$.

c Chemical-shift difference; $\Delta \delta=\delta(5-\mathrm{Na})-\delta(4-$ $\mathrm{Na}$.

(p. 94 footnote)

* Molecular peak $(1,016)$ for $\mathrm{C}_{54} \mathrm{H}_{89} \mathrm{O}_{16} \mathrm{Na}$ was observed in the EI-MS of 1-Na by Mr. OCCOLOwITZ of the Lilly Research Laboratories. Details will be published by him. 
As mentioned above, 2 is composed of 47 carbon atoms and the classification of the carbons, shown in Table 1, suggests that $\mathbf{2}$ should be an isomer of $\mathbf{3}$. The comparison between the ${ }^{13} \mathrm{C}$ spectra of $2-\mathrm{Na}$ and 3-Na revealed chemical-shift changes of 7 carbons which are assigned to those on the E-ring. The changes including a remarkable upfield shift of the C-26 signal (see $*$ and $* *$ in Table 1 ) can be explained only by the structure in which the 28-Me of 2-Na is epimeric ( $\alpha$-axial conformation) to that of $\mathbf{3}$ Na. Further, in comparison of the ${ }^{1} \mathrm{H}$ NMR spec-
Fig. 1. IR spectra of sodium salts of A-130B (a) and A-130C (b) in $\mathrm{CHCl}_{3}$.
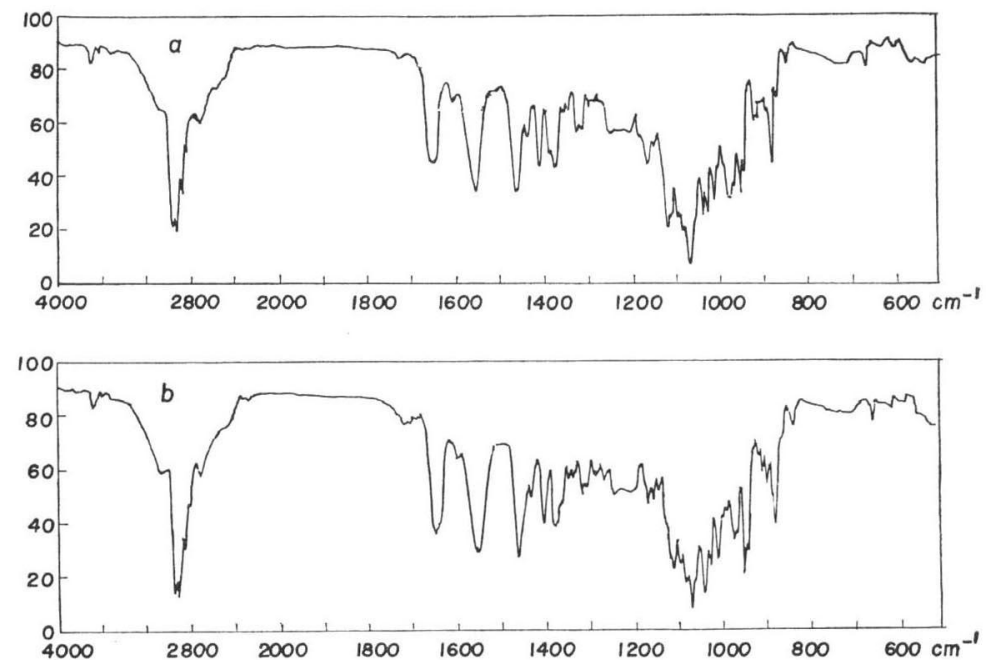

Fig. 2. ${ }^{1} \mathrm{H}$ NMR spectra of sodium salts of $\mathrm{A}-130 \mathrm{~B}$ (a) and $\mathrm{A}-130 \mathrm{C}$ (b) in $\mathrm{CDCl}_{3}$ (at $60 \mathrm{MHz}$ ).

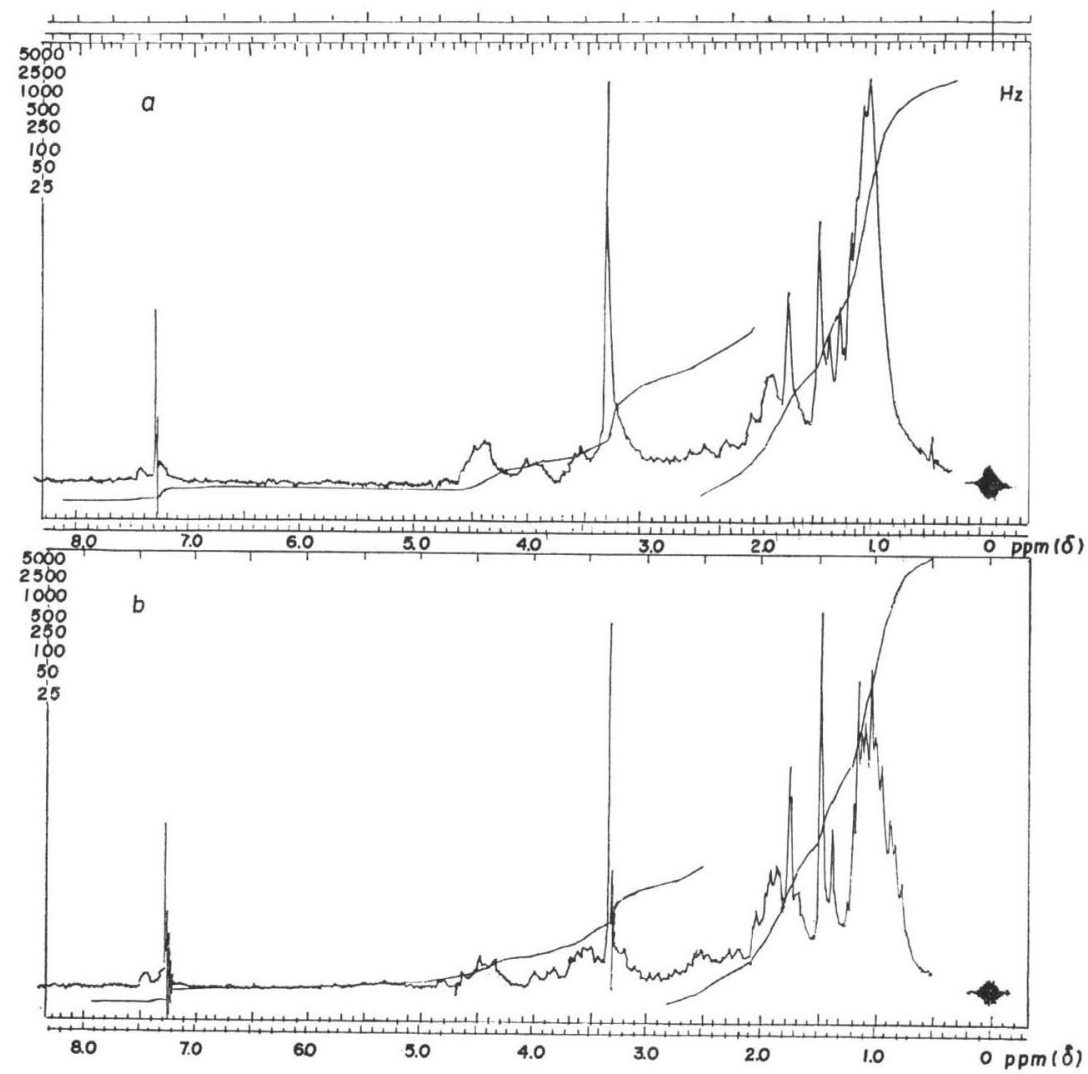


trum (in $\mathrm{C}_{6} \mathrm{D}_{6}$ ) of $2-\mathrm{Na}$ to that of $3-\mathrm{Na}$, one Me doublet moves downfield by $0.25 \mathrm{ppm}$ and another $\mathrm{Me}$ doublet upfield by $-0.06 \mathrm{ppm}$, while the other methyl signals are unchanged. This observation is in good agreement with the conclusion from ${ }^{13} \mathrm{C} \mathrm{NMR}$, and $\mathrm{A}-130 \mathrm{C}$ has been assessed to structure 2 .

A-130B is the second example of monovalent diglycoside polyether antibiotic (next to K-41B) according to WestLeY's classification ${ }^{3)}$ and A$130 \mathrm{C}$ is the first example of the polyether antibiotic having an axial methyl group on the "east" terminal ring.

The details of ${ }^{1} \mathrm{H}$ and ${ }^{18} \mathrm{C}$ NMR studies of A130 factors will be reported elsewhere in the near future ${ }^{11}$.

\section{Acknowledgments}

We thank Dr. R. L. Hamill of the Lilly Research Laboratories for providing us with the sample of 4-Na for ${ }^{13} \mathrm{C}$ NMR measurements.

\section{NAOKI TSUJI* \\ Yoshiniro TERUI \\ Kazuo Nagashima \\ KAZUO TORI}

Shionogi Research Laboratory,

Shionogi \& Co., Ltd.,

Fukushima-ku, Osaka, 553 Japan

\section{LeRoy F. JOHNSON}

Nicolet Technology Corporation 145 E. Dana Street, Mountain View, California, 94041 U.S.A.

(Received October 1, 1979)

\section{References}

1) Kubota, T.; H. Нinoh, M. Mayama, K. МотoKAWA \& Y. YASUDA: Antibiotic A-130, isolation and characterization. J. Antibiotics 28:
$931 \sim 934,1975$

2) a) Blount, J. F.; R. H. Evans, Jr., C.-M. Liu, T. Hermann \& J. W. Westley: X-Ray structure of Ro 21-6150, a polyether antibiotic related to dianemycin. J. Chem. Soc., Chem. Comm.-1975: 853 855, 1975

b) Koyama, H. \& K. Utsumi-Oda: Crystal and molecular structure of a silver salt of antibiotic A-130A. J. Chem. Soc., Perkin II 1977: $1531 \sim 1536,1977$

3) a) Westley, J. W.: Polyether antibiotics: Versatile carboxylic acid ionophores produced by Streptomyces. Adv. Applied Microbiol. 22: $177 \sim 223,1977$

b) Anteunis, M. J. O.; N. A. Rodios \& G. VERHEGGE: Solution conformation of dianemycin, its sodium salt and of lenoremycin- $\mathrm{Na}^{+}$ (Ro 21-6150 or A-130A). Bull. Soc. Chim. Belg. 86: 609 632, 1977

4) Hamill, R. L.; M. M. Hoehn, G. E. Pittenger, J. Chamberlin \& M. Gorman: Dianemycin, an antibiotic of the group affecting ion transport. J. Antibiotics 22: 161 164, 1969

5) WehrLI, F. W. \& T. Wirthlin: Interpretation of carbon-13 NMR spectra. Heyden, London, 1976

6) Eli Lilly Co.: U.S. Patent 3839560, 1974

7) Petcher, T. J. \& H.-P. Weber: X-Ray crystal structure and absolute configuration of $p$ bromo-phenacylseptamycin monohydrate, a polyether antibiotic. J. Chem. Soc., Chem. Comm. 1974: 697 698, 1974

8) Shiro, M.; H. Nakai, K. Nagashima \& N. TsuJI: X-Ray determination of the structure of the polyether antibiotic K-41. J. Chem. Soc., Chem. Comm. 1978: 682 683, 1978

9) Tsuji, N.; K. Nagashima, Y. Terui \& K. Tori: Structure of K-41B, a new diglycoside polyether antibiotic. J. Antibiotics 32: 169 172, 1979

10) Dorman, E. E.; R. L. Hamill, J. L. OCcolowitz, Y. Terui, K. Tori \& N. Tsuji, to be published

11) Terui, Y.; N. Tsuji, K. Nagashima, K. Tori \& L. F. Johnson, to be published 